\title{
Use of stellate ganglion block for treatment of recurrent syncope followed by chest pain
}

\author{
Young-ung Kim, Yong-joon Shin, Young Woo Cho \\ Department of Anesthesiology and Pain Medicine, Ulsan University Hospital, Ulsan, Korea
}

\begin{abstract}
Syncope is defined as a transient loss of consciousness and postural tone, characterized by rapid onset, short duration, and spontaneous recovery. Stellate ganglion block (SGB) is a nerve block method that is used for treatment of neuropathic pain in the head, neck and upper extremities, especially trigeminal neuralgia, postherpetic neuralgia and complex regional pain syndrome. SGB can modulate and stabilize the sympathetic nervous system, which prevents it from overexcitation and improves symptoms of syncope. The authors report a patient who was treated for pain and edema of both upper extremities with SGB, then showed improvement in recurrent syncope followed by chest pain and overall quality of life.
\end{abstract}

Keywords: Stellate ganglion block; Syncope; Chest pain; Upper extremity pain; Sympathetic nervous system

\section{INTRODUCTION}

Stellate ganglion block (SGB) is a selective sympathetic block that is widely used for neuropathic pain treatment. This procedure is used locally for disorders of the head and neck or upper extremities, especially trigeminal neuralgia, postherpetic neuralgia, complex regional pain syndrome (CRPS) and posttraumatic stress disorder (PTSD), as well as systemically for angina pectoris, psychosomatic disorder, hormonal disorders, or unbalanced sympathetic nervous system disorders [1].

Syncope is a transient loss of consciousness caused by transient global cerebral hypoperfusion that is characterized by rapid onset, short duration, and spontaneous complete recovery. Recovery from syncope is usually accompanied by almost immediate restoration of appropriate behavior and ori-

Received: August 3, 2017, Revised: September 22, 2017 Accepted: September 28, 2017

Corresponding Author: Young Woo Cho, Department of Anesthesiology and Pain Medicine, Ulsan University Hospital, 877, Bangeojinsunhwando-ro, Dong-gu, Ulsan 44033, Korea Tel: +82-52-250-7243, Fax: +82-52-250-7249

E-mail: ywcho67@gmail.com entation. Sometimes the post-recovery period may be marked by fatigue [2].

We report the case of a patient, who was treated for pain and edema of both upper extremities with SGB, then showed improvement in recurrent syncope followed by chest pain, as well as improved quality of life.

\section{CASE}

The 54-year-old female patient had no underlying disease except a past medical history of hemithyroidectomy to treat thyroid cancer 4 years prior. Following hemithyroidectomy, she developed left forearm pain and gradually progressed to pain accompanied by coldness and edema of both forearms and hands. The patient visited our pain clinic to treat the upper extremity pain, and SGB was performed. We explained the concept of SGB to the patient, as well as its potential side effects, including Horner's syndrome, hoarseness because of recurrent laryngeal nerve block, ipsilateral upper extremity motor weakness caused by brachial plexus block, and phrenic nerve block. The patient was laid down in the supine position and a pillow was put under her shoulders for cervical exten-

Copyright (C) 2018 Yeungnam University College of Medicine

This is an Open Access article distributed under the terms of the Creative Commons Attribution Non-Commercial License (http://creativecommons.org/licenses/by-nc/4.0/) which permits unrestricted non-commercial use, distribution, and reproduction in any medium, provided the original work is properly cited. 

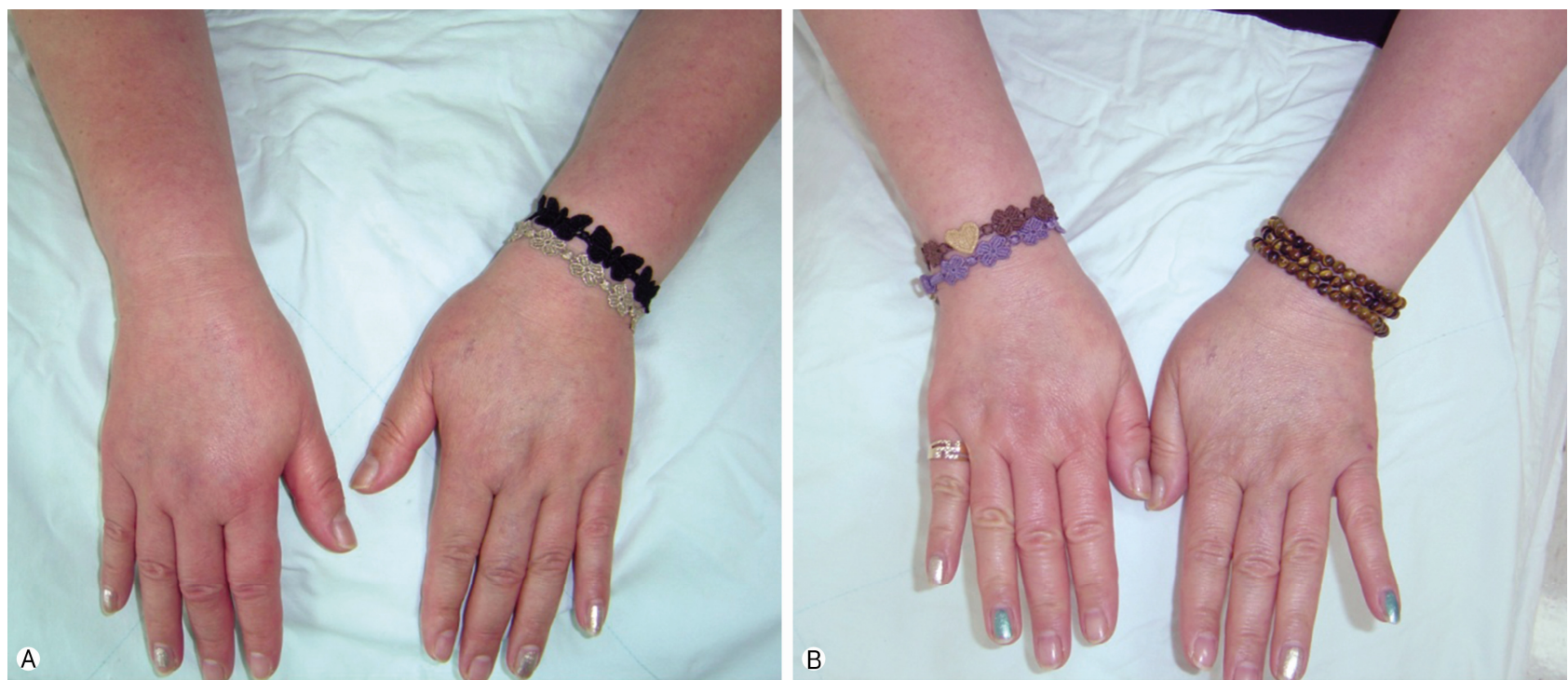

Fig. 1. (A) Photo of patient before treatment (SGB). Edema in both forearms and hands. (B) Photo of patient after treatment (SGB). Normalized both forearms and hands. SGB, stellate ganglion block.

sion to facilitate palpation of the bony landmarks. After preparing the skin, the needle was inserted blindly using an anterior paratracheal approach technique and advanced until it contacted the transverse process of the $\mathrm{C} 6$ vertebra. At that time, the needle was withdrawn slightly from the periosteum. After negative aspiration, $8 \mathrm{~mL}$ of $0.2 \%$ ropivacaine was slowly injected for SGB with repeated aspiration. Thereafter, the patient was observed for Horner's syndrome signs to confirm successful SGB.

The SGB was performed twice a week, alternating left and right. After 10 applications of SGB were performed, 5 times on each side, the numerical rating scale (NRS) score, which is an 11-point (0-10) scale for patient self-reporting of pain, decreased from 7 to 8 points to 2 to 3 points for both upper extremities, while the edema of both forearms and hands subsided almost completely (Fig. 1). We explained the successful progress of the treatment to the patient and recommended she reduce or discontinue further administration of SGB and continue treatment with oral medication. As oral medication, she was given milnacipran (Ixel) $12.5 \mathrm{mg}$ bid, which was quite effective on her upper extremity pain.

A month later, she mentioned that the pain in both arms got much better and interestingly, recurrent syncope with chest pain of unknown cause from about 13 years earlier also improved. Specifically, the frequency of syncope, which occurred more than once a day almost every day, was reduced to twice a week. Furthermore, the intensity of the preceding chest pain and time of loss of consciousness were also decreased. She said that the quality of life had improved and she was satisfied with this treatment effect.

Before trying SGB, whenever symptoms of syncope happened, the feature of the chest pain was a sharp pain of NRS 7 to 8 without radiating pain that lasted 10 minutes. When she came to the hospital, we were not able to keep track of all of her vital signs, but blood pressure, heart rate, respiratory rate, body temperature, and oxygen saturation were normal.

The patient said she underwent brain magnetic resonance imaging, electroencephalography, coronary angiography, tilt test, cardiac echography with ergonovine provocation test and Holter electrocardiography to find the cause of syncope followed by unexplained chest pain, but no abnormal findings were found. In addition, her electrocardiography has been monitored for 2 years using an implanted loop recorder, but no abnormal findings were observed, even when symptoms developed.

During the past several years, she has visited various medical departments (cardiovascular medicine, neurology, nephrology, allergy medicine, etc.) where she was given various tests and medications (vasoconstrictor, beta-blocker, SSRI, painkiller, anti-histamine, adrenal corticosteroid, etc.), but her symptoms did not improve significantly. Moreover, there were no underlying diseases observed during those visits. 
The cardiac autonomic neuropathy test (CAN) can be useful for assessing CAN simply, quickly and noninvasively and includes a test for heart rate variability, such as beat-beat variations with deep breathing, as well as changes on postural change from lying to standing and in response to the Valsalva maneuver. While the tests mentioned above revealed no abnormal findings, the CAN test confirmed cardiovascular autonomic abnormalities [3,4]. Therefore, we assumed that additional SGB would work and conducted it at the patient's request.

The SGB was performed twice a week, alternating left and right. After 20 applications of the treatment (10 on each side), the frequency of syncope decreased by 1-2 times a month. Before SGB was implemented, it was almost impossible for the patient to have a social life because of the syncope, but after SGB it became possible for her to have a social life and even travel. Additionally, she was very satisfied with the improved quality of life after treatment.

During SGB treatment, we recommended she try psychiatric treatment to check for psychiatric problems such as depression or PTSD, but she refused to do so. After about 3 months, she visited the hospital with both upper extremity pain that was slightly aggravated and requested additional SGB. After several SGB treatments, her pain improved gradually.

The syncope occurred irregularly once several months after the SGB stopped, but there was no preceding chest pain and the duration of syncope was shortened. At the time that this paper was written, she had experienced no additional syncope for almost a year after the last syncope, except for one or two experiences of loss of consciousness for a few seconds. The patient and her family were satisfied with her quality of life after treatment.

\section{DISCUSSION}

The mechanism of action of the SGB is not completely understood, but it has been widely used to treat sympathetically maintained pain, vascular disease and CRPS involving the face and upper arms, such as migraines, trigeminal neuralgia, atypical facial pain, hot flashes in postmenopausal women, PTSD and postherpetic neuralgia [5-7].

Syncope is a transient loss of consciousness caused by transient global cerebral hypoperfusion. The causes of syncope vary, but can be categorized into three groups; reflex syn- cope, syncope due to orthostatic hypotension and cardiac syncope [8]. Reflex syncope can be further divided into three types; vasovagal, situational, and carotid sinus syncope. Vasovagal syncope is mediated by either emotional distress (fear, pain, instrumentation, and blood phobia) or orthostatic stress. Situational syncope happens occurs in response to coughing, sneezing, gastrointestinal stimulation, micturition, exercise, eating, laughing, and etc. Carotid sinus syncope is triggered by mechanical manipulation of the carotid sinuses. Syncope due to orthostatic hypotension can be divided into four types; primary autonomic failure (pure autonomic failure, multiple system atrophy, Parkinson's disease with autonomic failure, Lewy body dementia), secondary autonomic failure (diabetes, amyloidosis, uremia, spinal cord injuries), drug-induced orthostatic hypotension, and syncope by volume depletion. Cardiac syncope can be divided into two types, those caused by arrhythmias and those caused by cardiac structural disease.

The patient in this case showed no particular disorders in the heart and brain related tests. However, the CAN test showed that it might have been caused by abnormal response of the autonomic nervous system in the cardiovascular system. After the patient received SGB, the symptoms of syncope improved; therefore, we considered that the abnormal results of the CAN test and the improvement in symptoms of syncope due to SGB may have been related to the autonomic nervous system as the cause of the patient's syncope. We recommended that the CAN test be conducted again to see if the results changed after SGB treatments, but the patient refused.

As mentioned above, the causes of syncope vary, and not all mechanism are clearly defined. However, syncope can be related either directly or indirectly to the autonomic nerve system, and this kind of syncope can be treated with SGB.

The role of the sympathetic nervous system, which is part of the autonomic nervous system, is to mobilize body resources under stress and to induce the fight-or-flight response. The sympathetic nervous system also remains constantly active at a basal level to maintain homeostasis. SGB can modulate the sympathetic nervous system [9].

The sympathetic nervous system controls cardiovascular function. Therefore, failure or overexcitation of the sympathetic nervous system causes disorder of cardiovascular function, which results in syncope. SGB may be beneficial to cardiovascular functions by regulating cardiac sympathetic nerv- 
ous functions without affecting hemodynamics [10]. SGB may also inhibit the increase of cardiac sympathetic nerve excitability induced by the excitation of cardiopulmonary baroreceptors $[10,11]$. If SGB can modulate the sympathetic nervous system, it can normalize the function of the cardiovascular autonomic nervous system and be effective on syncope. Therefore, we have come to believe that SGB has a prophylactic effect on syncope in patients who have autonomic impairment, as in this case.

In addition, the use of SGB to improve cerebral blood flow for treatment of cerebrovascular events is not new [12]. The effects of SGB include a significant increase in cerebral perfusion pressure because of a decrease in cerebrovascular tone [13]. We considered that, because syncope is mainly caused by global cerebral hypoperfusion, SGB can improve the symptoms of syncope by improving cerebral perfusion.

As we have already classified the causes of syncope above, pain was one of the reasons of syncope [8]. SGB is a common treatment for pain control that is used for treatment of neuropathic pain in the head, neck and upper extremity, especially trigeminal neuralgia, postherpetic neuralgia and CRPS. SGB can also be used to treat chest pain [14]. In the case of this patient, she had syncope followed by chest pain. The sympathetic nervous system can be stimulated by pain, which can affect cardiovascular autonomic function and cause syncope. Because SGB reduced chest pain, this resulted in decreased stimulation of the sympathetic nervous system, which may explain why the patient showed improved syncope.

There are a variety of treatments to manage syncope, including education, physical counter pressure maneuvers, tilt training, pharmacological therapy, and cardiac pacing [8]. Although we searched for treatments for syncope, reports of its treatment with SGB were rare. Although additional studies are needed, the present report is meaningful because it suggests that SGB can be used to treat syncope.

Although we cannot conclude that SGB is effective at treating syncope based on this single case, the findings presented in this study do indicate that SGB could be considered as a treatment for syncope when patients show no improvement of symptoms after treatment with existing methods. If there are more cases showing successful treatment of patients with syncope by SGB, its use will likely increase.

Overall, because of the improved prognosis and quality of life observed in the present study, future studies and trials investigating application of SGB to syncope are needed.

\section{CONFLICT OF INTEREST}

No potential conflict of interest relevant to this article was reported.

\section{ORCID}

Young Woo Cho, https://orcid.org/0000-0001-9683-1367

Young Ung Kim, https://orcid.org/0000-0002-6185-8786

\section{REFERENCES}

1. Wakusugi B. New application of stellate ganglion block. J Korean Pain Soc 1991;4:1-7. Korean.

2. Hoefnagels WA, Padberg GW, Overweg J, van der Velde EA, Roos RA. Transient loss of consciousness: the value of the history for distinguishing seizure from syncope. J Neurol 1991; 238:39-43.

3. Vinik AI, Maser RE, Mitchell BD, Freeman R. Diabetic autonomic neuropathy. Diabetes Care 2003;26:1553-79.

4. Yun JS, Ko SH, Ko SH, Song KH, Yoo KD, Yoon KH, et al. Cardiovascular disease predicts severe hypoglycemia in patients with type 2 diabetes. Diabetes Metab J 2015;39:498506.

5. Ackerman WE, Zhang JM. Efficacy of stellate ganglion blockade for the management of type 1 complex regional pain syndrome. South Med J 2006;99:1084-8.

6. Lipov EG, Joshi JR, Sanders S, Slavin KV. A unifying theory linking the prolonged efficacy of the stellate ganglion block for the treatment of chronic regional pain syndrome (CRPS), hot flashes, and posttraumatic stress disorder (PTSD). Med Hypotheses 2009;72:657-61.

7. Makharita MY, Amr YM, El-Bayoumy Y. Effect of early stellate ganglion blockade for facial pain from acute herpes zoster and incidence of postherpetic neuralgia. Pain Physician 2012; 15:467-74.

8. Task Force for the Diagnosis and Management of Syncope of the European Society of Cardiology (ESC); European Heart Rhythm Association (EHRA); Heart Failure Association (HFA); Heart Rhythm Society (HRS); Moya A, Sutton R, et al. Guidelines for the diagnosis and management of syncope (version 2009). Eur Heart J 2009;30:2631-71.

9. Lipov E. Post Traumatic Stress Disorder (PTSD) as an over activation of sympathetic nervous system: an alternative view. J Trauma Treat 2013;3:181.

10. Iwase $S$, Nishimura N, Mano T. Role of sympathetic nerve activity in the process of fainting. Front Physiol 2014;5:343.

11. Chen YQ Jin XJ, Liu ZF, Zhu MF. Effects of stellate ganglion block on cardiovascular reaction and heart rate variability in elderly patients during anesthesia induction and endotracheal intubation. J Clin Anesth 2015;27:140-5. 
12. Moore DC. Stellate ganglion block-therapy for cerebral vascular accidents. Br J Anaesth 2006;96:666

13. Gupta MM, Bithal PK, Dash HH, Chaturvedi A, Mahajan RP. Effects of stellate ganglion block on cerebral haemodynamics as assessed by transcranial Doppler ultrasonography.
Br J Anaesth 2005;95:669-73.

14. Parris WC, Lin S, Frist W Jr. Use of stellate ganglion blocks for chronic chest pain associated with primary pulmonary hypertension. Anesth Analg 1988;67:993-5. 